\title{
A new subclass of meromorphic functions with positive coefficients defining by linear operator
}

\author{
P. THIRUPATHI REDDY, B. VENKATESWARLU AND S. SREELAKSHMI
}

\begin{abstract}
In this paper, we introduce and study a new class $\sigma,(\alpha, \lambda)$ of meromorphic univalent functions defined in $E=\{z: z \in \mathbb{C}$ and $0<|z|<1\}=E \backslash\{0\}$. We obtain coefficient inequalities, distortion theorems, extreme points, closure theorems, radius of convexity estimates and integral operators. Finally, we obtained neighbourhood result for the class $\sigma_{p}(\gamma, \lambda)$.
\end{abstract}

Mathematics Subject Classification 2010: $30 \mathrm{C} 45$.

Keywords: meromorphic functions, analytic functions, neighborhood.

\section{INTRODUCTION}

Let $\Sigma$ denote the class of meromorphic functions of the form

$$
f(z)=\frac{1}{z}+\sum_{n=1}^{\infty} a_{n} z^{n},\left(a_{n} \geq 0\right)
$$

which are analytic in the punctured unit disc $E=\{z: z \in C$ and $0<|z|<1\}$. A function $f \in \sum$ is meromorphic starlike of order $\alpha(0 \leq \alpha<1)$ if

$$
-\operatorname{Re}\left\{\frac{z f^{\prime}(z)}{f(z)}\right\}>\alpha, \quad(z \in E) .
$$

The class of all such functions is denoted by $\Sigma^{*}(\alpha)$. A function $f \in \Sigma$ is meromorphic convex of order $\alpha \quad(0 \leq \alpha<1)$ if

$$
-\operatorname{Re}\left\{1+\frac{z f^{\prime \prime}(z)}{f^{\prime}(z)}\right\}>\alpha \quad(z \in E) .
$$

The class of such functions is denoted by $\Sigma_{k}^{*}(\alpha)$. The class $\Sigma(\alpha)$ and $\Sigma_{k}^{*}(\alpha)$ were introduced and studied by Clunie [1], Pommerenke [6], Miller [4] and Mogra et al. [5]. Let $\Sigma_{p}$ be the class of functions $f \in \Sigma$ with $a_{n} \geq 0$. The subclass of $\Sigma_{p}$ consisting of starlike function of order $\alpha$ is denoted by $\Sigma_{p}^{*}(\alpha)$. For a function $f(z) \in \Sigma$, Frasin 
and Darus [2] defined an operator $I^{k}: \Sigma \rightarrow \Sigma$ as follows

$$
\begin{aligned}
& I^{0} f(z)=f(z) \\
& I^{1} f(z)=z f^{\prime}(z)+\frac{2}{z} \\
& I^{2} f(z)=z\left(I^{1} f(z)\right)^{\prime}+\frac{2}{z}
\end{aligned}
$$

and for $k=1,2,3, \cdots$, we have

$$
\begin{aligned}
I^{k} f(z) & =z\left(I^{k-1} f(z)\right)^{\prime}+\frac{2}{z} \\
& =\frac{1}{z}+\sum_{n=1}^{\infty} n^{k} a_{n} z^{k} \quad\left(k \in N_{0}=N \cup\{0\}, z \in E\right) .
\end{aligned}
$$

Definition 1.1. Let $0 \leq \alpha<1$. Further let $f(z) \in \Sigma_{p}$ be given by (1), $0 \leq \lambda<1$. The class $\sigma_{p}(\alpha, \lambda)$ is defined by

$$
\sigma_{p}(\alpha, \lambda)=\left\{f \in \Sigma_{p}: \operatorname{Re}\left[\frac{z f^{\prime}(z)}{(\lambda-1) f(z)+\lambda z f^{\prime}(z)}\right]>\alpha\right\} .
$$

Clearly $\sigma_{p}(\alpha, 0)$ reduces to $\Sigma_{p}^{*}(\alpha)$.

In this paper, we obtain the coefficient inequalities, growth and distortion inequalities, as well as closure results for the class $\sigma_{p}(\alpha, \lambda)$. Properties of certain integral operators and neighbourhood properties are also discussed for the class. The subclass of $\Sigma_{p}$ consisting of starlike functions of order $\alpha$ is denoted by $\Sigma_{p}^{*}(\alpha)$.

\section{COEFFICIENTS INEQUALITIES}

Our first theorem gives a necessary and sufficient condition for a function $f$ to be in the class $\Sigma_{p}(\lambda, \alpha)$.

THEOREM 2.1. Let $f(z) \in \Sigma_{p}$ be given by (1). Then $f \in \sigma_{p}(\lambda, \alpha)$ if and only if

$$
\sum_{n=1}^{\infty} n^{k}[n+\alpha-\alpha \lambda(1+n)] a_{n} \leq 1-\alpha
$$

Proof. If $f \in \sigma_{p}(\lambda, \alpha)$ then

$$
\operatorname{Re}\left\{\frac{z\left(I^{k} f(z)\right)^{\prime}}{(\lambda-1)\left(I^{k} f(z)\right)+\lambda z\left(I^{k} f(z)\right)^{\prime}}\right\}=\operatorname{Re}\left\{\frac{-1+\sum_{n=1}^{\infty} n^{k+1} a_{n} z^{n+1}}{-1+\sum_{n=1}^{\infty}(\lambda-1+\lambda n) n^{k} a_{n} z^{n+1}}\right\}>\alpha .
$$


By letting $z \rightarrow 1^{-}$, we have $\left\{\frac{-1+\sum_{n=1}^{\infty} n^{k+1} a_{n}}{-1+\sum_{n=1}^{\infty} n^{k}(\lambda-1+\lambda n) a_{n}}\right\}>\alpha$.

This shows that (5) holds.

Conversely assume that (5) holds. Since

$\operatorname{Re}(w)>\alpha$ if and only if $|w-1|<|w+1-2 \alpha|$.

It is sufficient to show that

$$
\left|\frac{z\left(I^{k} f(z)\right)^{\prime}-\left[(\lambda-1) I^{k} f(z)+\lambda z\left(I^{k} f(z)\right)^{\prime}\right]}{z\left(I^{k} f(z)\right)^{\prime}+(1-2 \alpha)\left[(\lambda-1) I^{k} f(z)+\lambda z\left(I^{k} f(z)\right)^{\prime}\right]}\right|<1 .
$$

Using (5), we see that

$$
\begin{aligned}
& \left|\frac{z\left(I^{k} f(z)\right)^{\prime}-\left[(\lambda-1) I^{k} f(z)+\lambda z\left(I^{k} f(z)\right)^{\prime}\right]}{z\left(I^{k} f(z)\right)^{\prime}+(1-2 \alpha)\left[(\lambda-1) I^{k} f(z)+\lambda z\left(I^{k} f(z)\right)^{\prime}\right]}\right| \\
= & \left|\frac{\sum_{n=1}^{\infty} n^{k}(1-\lambda)(n+1) a_{n} z^{n+1}}{-2(1-\alpha)+\sum_{n=1}^{\infty} n^{k}[n(1+(1-2 \alpha) \lambda)+(1-2 \alpha)(\lambda-1)] a_{n} z^{n+1}}\right| \\
\leq & \frac{\sum_{n=1}^{\infty} n^{k}(1-\lambda)(n+1) a_{n}}{2(1-\alpha)-\sum_{n=1}^{\infty} n^{k}[n(1+(1-2 \alpha) \lambda)+(1-2 \alpha)(\lambda-1)] a_{n}} \\
\leq & 1 .
\end{aligned}
$$

Thus we have $f \in \sigma_{p}(\lambda, \alpha)$.

Corollary 2.2. If $f \in \sigma_{p}(\lambda, \alpha)$ then

$$
a_{n} \leq \frac{(1-\alpha)}{n^{k}[n+\alpha-\alpha \lambda(1+n)]} .
$$

PROOF. The result is sharp for the functions $F_{n}(z)$ given by

$$
F_{n}(z)=\frac{1}{z}+\frac{(1-\alpha)}{n^{k}[n+\alpha-\alpha \lambda(1+n)]} z^{n}, \quad n=1,2,3 \cdots .
$$

\section{GROWTH AND DISTORTION THEOREM}

THEOREM 3.1. If $f \in \sigma_{p}(\lambda, \alpha)$, then

$$
\frac{1}{r}-\frac{(1-\alpha)}{(1+\alpha-2 \alpha \lambda)} r \leq|f(z)| \leq \frac{1}{r}+\frac{(1-\alpha)}{(1+\alpha-2 \alpha \lambda)} r .
$$


The result is sharp for the function

$$
f(z)=\frac{1}{z}+\frac{1-\alpha}{1+\alpha-2 \alpha \lambda} z .
$$

ProOF. Since $f(z)=\frac{1}{z}+\sum_{n=1}^{\infty} a_{n} z^{n}$, we have

$$
|f(z)| \leq \frac{1}{r}+\sum_{n=1}^{\infty} a_{n} r^{n} \leq \frac{1}{r}+r \sum_{n=1}^{\infty} a_{n}
$$

since,

$$
\sum_{n=1}^{\infty} a_{n} \leq \frac{1-\alpha}{1+\alpha-2 \alpha \lambda}
$$

Using this, we have

$$
|f(z)| \leq \frac{1}{r}+\frac{1-\alpha}{1+\alpha-2 \alpha \lambda} r
$$

Similarly

$$
|f(z)| \geq \frac{1}{r}-\frac{1-\alpha}{1+\alpha-2 \alpha \lambda} r .
$$

The result is sharp for $f(z)=\frac{1}{z}+\frac{1-\alpha}{1+\alpha-2 \alpha \lambda} z$.

THEOREM 3.2. If $f \in \sigma_{p}(\alpha, \lambda)$ then

$$
\frac{1}{r^{2}}-\frac{1-\alpha}{1+\alpha-2 \alpha \lambda} \leq\left|f^{\prime}(z)\right| \leq \frac{1}{r^{2}}+\frac{1-\alpha}{1+\alpha-2 \alpha \lambda} \quad(|z|=r) .
$$

The result is sharp for the function given by (7).

\section{CLOSURE THEOREMS}

Let the functions $F_{k}(z)$ be given by

$$
F_{k}(z)=\frac{1}{z}+\sum_{n=1}^{\infty} a_{n, k} z^{n}, \quad k=1,2,3, \cdots .
$$

We shall prove the following closure theorems for the class $\sigma_{p}(\lambda, \alpha)$.

THEOREM 4.1. Let the function defined by (8) be in the class $\sigma_{p}(\lambda, \alpha)$ for every $k=1,2,3, \cdots$. Then the function $f(z)$ defined by

$$
f(z)=\frac{1}{z}+\sum_{n=1}^{\infty} a_{n} z^{n}, \quad\left(a_{n} \geq 0\right)
$$


belongs to the class $\sigma_{p}(\lambda, \alpha)$, where $a_{n}=\frac{1}{m} \sum_{n=1}^{m} a_{n, k}(n=1,2, \cdots)$.

ProOF. Since $F_{k}(z) \in \sigma_{p}(\lambda, \alpha)$, it follows from Theorem 2.1 that,

$$
\sum_{n=1}^{\infty} n^{k}[n+\alpha-\alpha \lambda(1+n)] a_{n, k} \leq 1-\alpha, \text { for all } k=1,2, \cdots, m .
$$

Hence

$$
\begin{aligned}
& \sum_{n=1}^{\infty} n^{k}[n+\alpha-\alpha \lambda(1+n)] a_{n} \\
= & \sum_{n=1}^{\infty} n^{k}[n+\alpha-\alpha \lambda(1+n)]\left(\frac{1}{m} \sum_{k=1}^{m} a_{n, k}\right) \\
= & \frac{1}{m} \sum_{n=1}^{m}\left(\sum_{n=1}^{\infty} n^{k}[n+\alpha-\alpha \lambda(1+n)] a_{n, k}\right) \\
& \leq 1-\alpha .
\end{aligned}
$$

By Theorem 2.1, we have $f(z) \in \sigma_{p}(\lambda, \alpha)$.

TheOREM 4.2. The class $\sigma_{p}(\alpha, \lambda)$ is closed under convex linear combination.

Proof. Let the function $F_{k}(z)$ given by (8) be in the class $\sigma_{p}(\alpha, \lambda)$. Then it is enough to show that the function

$$
H(z)=\lambda F_{1}(z)+(1-\lambda) F_{2}(z), \quad(0 \leq \lambda \leq 1)
$$

is also in the class $\sigma_{p}(\alpha, \lambda)$. Since for $(0 \leq \lambda \leq 1)$,

$$
H(z)=\frac{1}{z}+\sum_{n=1}^{\infty}\left[\lambda a_{n, 1}+(1-\lambda) a_{n, 2}\right] z^{n} .
$$

We observe that

$$
\begin{aligned}
& \sum_{n=1}^{\infty}\left\{n^{k}[n+\alpha-\alpha \lambda(1+n)]\right\}\left[\lambda a_{n, 1}+(1-\lambda) a_{n, 2}\right] \\
& \quad=\lambda \sum_{n=1}^{\infty}\left\{n^{k}[n+\alpha-\alpha \lambda(1+n)]\right\} a_{n, 1}+(1-\lambda) \sum_{n=1}^{\infty}\left\{n^{k}[n+\alpha-\alpha \lambda(1+n)]\right\} a_{n, 2} \\
& \quad \leq 1-\alpha .
\end{aligned}
$$

By Theorem 2.1, we have $H(z) \in \sigma_{p}(\alpha, \lambda)$.

THEOREM 4.3. Let $F_{0}(z)=1$ and

$$
F_{n}(z)=\frac{1}{z}+\sum_{n=1}^{\infty} \frac{1-\alpha}{n^{k}[n+\alpha-\alpha \lambda(1+n)]} z^{n}, \text { for } n=1,2, \cdots .
$$


Then $f(z) \in \sigma_{p}(\alpha, \lambda)$ if and only if $f(z)$ can be expressed in the form $f(z)=\sum_{n=0}^{\infty} \lambda_{n} F_{n}(z)$, where $\lambda_{n} \geq 0$ and $\sum_{n=0}^{\infty} \lambda_{n}=1$.

PROOF. Let

$$
\begin{aligned}
f(z) & =\sum_{n=0}^{\infty} \lambda_{n} F_{n}(z) \\
& =\frac{1}{z}+\sum_{n=1}^{\infty} \frac{\lambda_{n}(1-\alpha)}{n^{k}[n+\alpha-\alpha \lambda(1+n)]} z^{n} .
\end{aligned}
$$

Then

$$
\begin{aligned}
& \sum_{n=1}^{\infty} \frac{\lambda_{n}(1-\alpha)}{n^{k}[n+\alpha-\alpha \lambda(1+n)]} \frac{n^{k}[n+\alpha-\alpha \lambda(1+n)]}{(1-\alpha)} \\
= & \sum_{n=1}^{\infty} \lambda_{n}=1-\lambda_{0} \leq 1 .
\end{aligned}
$$

By Theorem 2.1, we have $H(z) \in \sigma_{p}(\alpha, \lambda)$.

Conversely, let $f(z) \in \sigma_{p}(\alpha, \lambda)$. From Theorem 2.1, we have

$$
a_{n} \leq \frac{1-\alpha}{n^{k}[n+\alpha-\alpha \lambda(1+n)]} \text { for } n=1,2, \cdots .
$$

We may take $\lambda_{n}=\frac{n^{k}[n+\alpha-\alpha \lambda(1+n)]}{1-\alpha} a_{n}$ for $n=1,2, \cdots$ and $\lambda_{0}=1-\sum_{n=1}^{\infty} \lambda_{n}$.

Then $f(z)=\sum_{n=0}^{\infty} \lambda_{n} F_{n}(z)$.

\section{RADIUS OF MEROMORPHIC STARLIKENESS AND MEROMORPHIC CONVEXITY}

THEOREM 5.1. Let the function $f$ be in the class $\sigma_{p}(\alpha, \lambda)$. Then $f$ is meromorphically starlike of order $\rho(0 \leq \rho<1)$ in $|z|<r_{1}(\alpha, \lambda, \rho)$, where

$$
r_{1}(\alpha, \lambda, \rho)=\inf _{n \geq 1}\left[\frac{(1-\rho)(1-\alpha)}{n^{k}(n+2-\rho)[n+\alpha-\alpha \lambda(1+n)]}\right]^{\frac{1}{n+1}}
$$

PROOF. Let $f(z)$ is in $\sigma_{p}(\alpha, \lambda)$. Then by Theorem 2.1, we have

$$
n^{k}[n+\alpha-\alpha \lambda(1+n)] a_{n} \leq(1-\alpha) .
$$

It is sufficient to show that

$$
\left|1+\frac{z f^{\prime}(z)}{f(z}\right| \leq 1-\rho
$$


Or equivalently

$$
\left|1+\frac{z f^{\prime}(z)}{f(z)}\right|=\left|\frac{\sum_{n=1}^{\infty}(n+1) a_{n} z^{n}}{\frac{1}{z}-\sum_{n=1}^{\infty} a_{n} z^{n}}\right| \leq 1-\rho
$$

or

$$
\sum_{n=1}^{\infty} \frac{(n+2-\rho)}{1-\rho} a_{n}|z|^{n+1} \leq 1, \text { for } 0 \leq \rho<1 \text { and }|z|<r_{1}(\alpha, \lambda, \rho) .
$$

By theorem 2.1, (12) will be true if

$$
\left(\frac{n+2-\rho}{1-\rho}\right)|z|^{n+1} \leq \frac{1-\alpha}{n^{k}[n+\alpha-\alpha \lambda(1+n)]}
$$

or if

$$
|z| \leq\left[\frac{1-\alpha}{(n+2-\rho)\left\{n^{k}[n+\alpha-\alpha \lambda(1+n)]\right\}}\right]^{\frac{1}{n+1}}, n \geq 1 .
$$

This completes the proof of Theorem.

THEOREM 5.2. Let the function $f(z)$ be in the class $\sigma_{p}(\alpha, \lambda)$. Then $f$ is meromorphically convex of order $\rho(0 \leq \rho<1)$ in $|z|<r_{2}(\alpha, \lambda, \rho)$, where

$$
r_{2}(\alpha, \lambda, \rho)=\inf _{n \geq 1}\left[\frac{(1-\rho)\left\{n^{k}[n+\alpha-\alpha \lambda(n+1)]\right\}}{n(n+2-\rho)(1-\alpha)}\right]^{\frac{1}{n+1}}, n \geq 1 .
$$

Proof. Let $f(z)$ be in $\sigma_{\rho}(\alpha, \lambda)$. Then by Theorem 2.1, we have

$$
\sum_{n=1}^{\infty} n^{k}[n+\alpha-\alpha \lambda(1+n)] a_{n} \leq(1-\alpha) .
$$

It is sufficient to show that

$$
\left|2+\frac{z f^{\prime \prime}(z)}{f^{\prime}(z}\right| \leq 1-\delta
$$

for $|z|<r_{2}=r_{2}(\alpha, \lambda, \rho)$, where $r_{2}(\alpha, \lambda, \rho)$ is specified in the statement of the Theorem. Then

$$
\left|2+\frac{z f^{\prime \prime}(z)}{f^{\prime}(z)}\right|=\left|\frac{\sum_{n=1}^{\infty} n(n+1) a_{n} z^{n-1}}{\frac{-1}{z^{2}}+\sum_{n=1}^{\infty} n a_{n} z^{n-1}}\right| \leq \frac{\sum_{n=1}^{\infty} n(n+1) a_{n}|z|^{n+1}}{1-\sum_{n=1}^{\infty} n a_{n}|z|^{n+1}} .
$$

This will be bounded by $(1-\rho)$ if

$$
\sum_{n=1}^{\infty} \frac{n(n+2-\delta)}{1-\delta} a_{n}|z|^{n+1} \leq 1
$$


By (14), it follows that (15) is true if

$$
\frac{n(n+2-\rho)}{1-\rho}|z|^{n+1} \leq \frac{n^{k}[n+\alpha-\alpha \lambda(1+n)]}{1-\alpha}, n \geq 1
$$

or

$$
|z| \leq\left[\frac{(1-\rho)\left\{n^{k}[n+\alpha-\alpha \lambda(1+n)]\right\}}{n(n+2-\rho)(1-\alpha)}\right]^{\frac{1}{n+1}}, n \geq 1 .
$$

This completes the proof of Theorem.

\section{INTEGRAL OPERATORS}

In this section, we consider integral transform of functions in the class $\sigma_{p}(\alpha, \lambda)$.

THEOREM 6.1. Let the function $f(z)$ given by (1) be in $\sigma_{p}(\alpha, \lambda)$. Then the integral operator

$$
F(z)=c \int_{0}^{1} u^{c} f(u z) d u(0 \leq u \leq 1,0<c<\infty)
$$

is in $\sigma_{p}(\delta, \lambda)$, where

$$
\delta=\frac{(c+2)(1+\alpha-2 \alpha \lambda)-c(1-\alpha)}{c(1-\alpha)(1-2 \lambda)+(1+\alpha)(1-2 \lambda)(c+2)} .
$$

The result is sharp for the function

$$
f(z)=\frac{1}{z}+\frac{1-\alpha}{(1+\alpha-2 \alpha \lambda)} z .
$$

Proof. Let $f(z) \in \sigma_{p}(\alpha, \lambda)$. Then

$$
\begin{aligned}
F(z) & =c \int_{0}^{1} u^{c} f(u z) d u \\
& =\frac{1}{z}+\sum_{n=1}^{\infty} \frac{c}{c+n+1} a_{n} z^{n} .
\end{aligned}
$$

It is sufficient to show that

$$
\sum_{n=1}^{\infty} \frac{c\left\{n^{k}[n+\delta-\delta \lambda(1+n)]\right\}}{(c+n+1)(1-\delta)} a_{n} \leq 1 .
$$

Since $f \in \sigma_{p}(\alpha, \lambda)$, we have

$$
\sum_{n=1}^{\infty} \frac{n^{k}[n+\alpha-\alpha \lambda(1+n)]}{(1-\alpha)} a_{n} \leq 1
$$


Note that (17) is satisfied if

$$
\frac{c[n+\delta-\delta \lambda(1+n)]}{(c+n+1)(1-\delta)} \leq \frac{[n+\alpha-\alpha \lambda(1+n)]}{(1-\alpha)} .
$$

Solving for $\delta$, we have

$$
\delta \leq \frac{(c+n+1)[n+\alpha-\alpha \lambda(1+n)]-c n(1-\alpha)}{c(1-\alpha)[1-\lambda(1+n)]+[n+\alpha-\alpha \lambda(1+n)](c+n+1)}=G(n) .
$$

A simple computation will show that $G(n)$ is increasing and $G(n) \geq G(1)$. Using this, the result follows.

For the choice of $\lambda=0$, we have the following result of Uralegaddi and Gangi [8].

Remark 6.2. Let the function $f(z)$ defined by (1) be in $\Sigma_{p}^{*}(\alpha)$. Then integral operator

$$
F(z)=c \int_{0}^{1} u^{c} f(u z) d u \quad(0<u \leq 1,0<c<\infty)
$$

is in $\Sigma_{p}^{*}(\alpha)$, where $\delta=\frac{1+\alpha+c \alpha}{1+\alpha+c}$.

The result is sharp for the function

$$
f(z)=\frac{1}{z}+\frac{1-\alpha}{1+\alpha} z
$$

\section{NEIGHBOURHOODS FOR THE CLASS $\Sigma_{P}^{\gamma}(\alpha, \lambda)$}

In this section, we determine the neighbourhood for the class $\Sigma_{p}^{\gamma}(\alpha, \lambda)$, which we define as follows.

Definition 7.1. A function $f \in \Sigma_{p}$ is said to be in the class $\sigma_{p}^{\gamma}(\alpha, \lambda)$ if there exists a function $g \in \sigma_{p}^{\gamma}(\alpha, \lambda)$ such that

$$
\left|\frac{f(z)}{g(z)}-1\right|<1-\gamma, \quad(z \in E, 0 \leq \gamma<1) .
$$

Following the earlier works on neighbourhoods of analytic functions by Goodman [3] and Ruscheweyh [7], we define the $\delta$-neighbourhood of function $f \in \Sigma_{p}$ by

$$
N_{\delta}(f)=\left\{g \in \Sigma_{p}: g(z)=\frac{1}{z}+\sum_{n=1}^{\infty} b_{n} z^{n} \text { and } \sum_{n=1}^{\infty} n\left|a_{n}-b_{n}\right| \leq \delta\right\} .
$$


THEOREM 7.2. If $g \in \sigma_{p}(\alpha, \lambda)$ and

$$
\gamma=1-\frac{\delta(1+\alpha-2 \alpha \lambda)}{2 \alpha-2 \alpha \lambda}
$$

then $N_{\delta}(g) \subset \sigma_{p}^{\gamma}(\alpha, \lambda)$.

Proof. Let $f \in N_{\delta}(g)$. Then we find from (20) that

$$
\sum_{n=1}^{\infty} n\left|a_{n}-b_{n}\right| \leq \delta
$$

which implies the coefficient inequality

$$
\sum_{n=1}^{\infty}\left|a_{n}-b_{n}\right| \geq \delta \quad(n \in N) .
$$

Since $g \in \sigma_{p}(\alpha, \lambda)$, we have

$$
\sum_{n=1}^{\infty} b_{n} \leq \frac{1-\alpha}{1+\alpha-2 \alpha \lambda} .
$$

So that

$$
\begin{aligned}
\left|\frac{f(z)}{g(z)}-1\right| & <\frac{\sum_{n=1}^{\infty}\left|a_{n}-b_{n}\right|}{1-\sum_{n=1}^{\infty} b_{n}} \\
& =\frac{\delta(1+\alpha-2 \alpha \lambda)}{2 \alpha-2 \alpha \lambda} \\
& =1-\gamma
\end{aligned}
$$

provided $\gamma$ is given by (21). Hence by definition, $f \in \sigma_{p}^{\gamma}(\alpha, \lambda)$.

\section{ACKNOWLEDGEMENTS}

The authors express their sincere thanks to the esteemed referee(s) for their careful readings, valuable suggestions and comments, which helped them to improve the presentation of the paper. 


\section{REFERENCES}

J. Clunie, On meromorphic schlicht functions, J. London Math. Soc., 34(1959), 215-216.

B.A. Frasin and M. Darus, On certain meromorphic functions with positive coefficients, South East Asian Bull. of Math., 28 (2004), 615-623.

A.W. Goodman, Univalent functions and non-analytic curves, Proc. Amer. Math. Soc., (1957), 598-601.

J. E. Miller, Convex meromorphic mappings and related functions, Proc. Amer. Math. Soc., 25 (1970), 220-228.

M. L. Mogra, T. R. Reddy and O. P. Juneja, Meromorphic univalent functions with positive coefficients, Bull. Aus. Math. Soc., 32(1985), 161-176.

Ch. Pommarenke, On meromorphic starlike functions, Pacfic J. Math., 13(1963), 221-235.

St. Ruscheweyh, Neighbourhoods of univalent functions, Proc. Amer. Math. Soc., 81 (1981), 521-527.

B. A. Uralegaddi and M. D. Gangi, A certain class of meromorphically starlike functions with positive coefficients, Pure Appl. Math. Sci., 26 (1987), 75-81.

P. Thirupathi Reddy

Department of Mathematics, Kakatiya Univeristy,

Warangal- 506 009, Telangana, India.

email: reddypt2@gmail.com

B. Venkateswarlu (Corresponding Author)

Department of Mathematics, GST, GITAM University

Doddaballapur- 561 203, Bengaluru Rural, India.

email: bvlmaths@gmail.com

\section{S. Sreelakshm}

Department of Mathematics, T S W R J College ,

Elkathurthy - 505 476, Warangal Urban, Telangana, India.

email: sreelakshmisarikonda@gmail.com 